\title{
EL DISCURSO SOBRE LA TRADUCCIÓN EN LA PRENSA ESPAÑOLA (2013-2017) LA TRADUCCIÓN COMO NOTICIA
}

\author{
(Discourse on translation in Spanish newspapers (2013-2017): translation as news)
}

Silvia Montero Küpper ${ }^{1}$

(Universidade de Vigo)

\begin{abstract}
RESUMEN
Utilizando una metodología descriptivo-cualitativa, en esta investigación se estudia de qué manera se presenta la traducción como noticia en un periódico de ámbito estatal (El País) y otro regional (La Voz de Galicia). Nos proponemos identificar y analizar el discurso sobre la traducción, su visibilidad y valoración. El corpus, que corresponde al lustro 2013-2017, se conforma de aquellos documentos en los que se registra la palabra traducción en el titular. Si bien el discurso sobre la traducción es mayoritariamente positivo, especialmente cuando se refiere a la traducción editorial, se constata la práctica ausencia de un discurso de la traducción periodística en los medios analizados.

Palabras clave: Traducción. Visibilidad de la traducción. Imagen de la traducción. Discurso sobre la traducción. Prensa española. Poder publicativo.

\section{RESUMO}

Utilizando uma metodologia descritivo-qualitativa, esta pesquisa estuda como a tradução é apresentada como notícia em jornais espanhóis de âmbito estadual (El País) e regional (La Voz de Galicia). Trata-se de identificar e analisar o discurso sobre a tradução, a sua visibilidade e valoração. O corpus, que corresponde a um período de cinco anos (2013-2017), é constituído por artigos em que a palavra 'tradução' faz parte da manchete. Embora o discurso sobre a tradução seja principalmente positivo, especialmente quando se refere à tradução editorial, constata-se, praticamente, a ausência de um discurso da tradução jornalística nas mídia analisadas.
\end{abstract}

Palavras chave: Tradução. Visibilidade da tradução. Imagem da tradução. Discurso sobre a tradução. Imprensa espanhola. Quarto poder.

\begin{abstract}
Using a descriptive-qualitative methodology, this paper studies how translation is presented as news in a national (El País) and a regional (La Voz de Galicia) newspaper. It aims at identifying and analysing the general discourse on translation, its visibility and valuation. The corpus comprises a period of five years (2013-2017) and is composed by articles which headlines register the word 'translation'. Although the discourse on translation is mainly positive, especially when it refers to editorial translation, it was found that a journalistic translation discourse in the analysed media is practically lacking.
\end{abstract}

Keywords: Translation. Visibility of translation. Image of translation. Translational discourse. Spanish news media. Fourth estate.

Recebido em: agosto 2019

Aceito em: dezembro 2019

DOI: $\underline{10.26512 / \text { les.v20i2.26546 }}$

\footnotetext{
1 M.A. en Filología Románica, Literatura Alemana Moderna y Literatura Comparada, doctora en Traducción e Interpretación; profesora del Dpto. de Traducción y Lingüística de la Universidade de Vigo y miembro titular del Grupo de Investigación en Estudos literarios e culturais, Tradución e Interpretación BiFeGa (Programa de Consolidación e Estruturación de Unidades de Investigación Competitivas, Ref. ED431B 2017176); líneas de investigación: traducción gallega, políticas de traducción editorial, discurso metatraductivo en la prensa; smontero@uvigo.es.
} 


\section{INTRODUCCIÓN}

El carácter globalizador que la información y la propia prensa adquieren, la opción discursivo-traductiva y las traducciones periodísticas en cuanto contenidos que se transmiten, conforman la visión y el entendimiento de los hechos, de la política o del mundo. No son pocas las voces que indican que la traducción periodística y, asimismo, la traducción en general, suelen pasar inadvertidas a pesar de ser "factor constitutivo [...] de la era transmoderna" (MARTÍN RUANO \& VIDAL SALES, 2013, p. 85-86). Sería deseable que hubiese comentarios metatraductivos en los propios medios de comunicación de manera implícita o explícita en forma de posicionamientos ideológicos o epistemológicos ${ }^{2}$.

El presente artículo pretende contribuir al estudio en este ámbito considerando la estima y el valor que se le otorga a la traducción, así como el lugar que esta ocupa en y para la prensa. Así, nos hemos propuesto como objetivo analizar el discurso explícito o subyacente sobre la traducción en los medios de comunicación y su visibilidad.

A partir de un corpus ad hoc, obtenido de artículos de dos periódicos publicados entre los años 2013 y 2017, analizamos los juicios emitidos sobre la traducción (periodística, editorial, audiovisual, etc.), así como el papel cuantitativo y cualitativo que esta desempeña como hecho noticioso. No focalizamos, por lo tanto, únicamente en la traducción periodística, entendida esta como proceso de transculturación inherente a la práctica profesional de las y los periodistas, también denominada ‘transedición' (STETTING, 1989)' ${ }^{3}$. No obstante, consideramos oportuno, prestar especial atención a la traducción periodística por ser altamente consustancial a los medios.

\section{Sobre La Traducción En Los Medios De Comunicación}

En los últimos 20 años, la investigación sobre la traducción en los medios de comunicación, sobre todo en la prensa, se ha intensificado y diversificado considerablemente. Los estudios en traducción y periodismo abordan los factores y las instancias que intervienen en la selección y conformación informativa desde varios planteamientos que aquí no podemos exponer ${ }^{4}$. Las publicaciones sobre el papel de la traducción y la ideología en un contexto de transmisión en el ámbito político también están ganando terreno (cf. BASTIN \& ITURRIZA, 2008; GAGNON, 2012;

\footnotetext{
${ }^{2}$ En lo que concierne a la traducción periodística, VALDEÓN (2016, p. 46) ofrece un ejemplo de posicionamiento ideológico de la BBC que esta explicita cuando justifica en su sitio web el uso de "separatistas" (versus "terroristas") para remitir a los integrantes del grupo armado ETA, incluso en las traducciones al inglés. No obstante, señalamos que el posicionamiento (implícito) dimana de las normas editoriales y estas no suelen acompañar a la noticia.

${ }^{3} C f$. también el concepto de journalator (VAN DOORSLAER, 2012) en referencia al periodista o traductor-periodista.

${ }^{4} C f$. VALDEÓN (2015) que ofrece un recorrido sobre la investigación en este ámbito desde principio de este siglo.
} 
VALDEÓN, 2012; ZANETTIN, 2016; MARTÍN RUANO \& VIDAL CLARAMONTE, 2016, etc.). La socióloga BIELSA, por ejemplo, postula una consciencia de la importancia de la traducción y la relaciona con una disposición cosmopolita (2016, p. 32). Por esta razón consideramos preciso insistir en el papel de la traducción, y no solo de la traducción periodística, en la sociedad a nivel local y global, tanto en lo que afecta el ámbito privado, cultural, económico como el político. Esto nos ha llevado a iniciar un trabajo que contempla el discurso sobre la traducción en la prensa como poderosa institución que debe velar por una información fiable y contrastada. Mencionamos, en este contexto, únicamente la capacidad que ostenta la traducción para manipular o globalizar conceptos ${ }^{5}$. Subrayamos, igualmente, la responsabilidad que tiene el 'poder publicativo' para con la sociedad, que no implica la mera información, sino, asimismo, la formación, a la vez que conlleva la creación de opinión pública, nunca exenta de ideología.

En los estudios sobre traducción periodística resulta recurrente la insistencia en la invisibilidad de la traducción en los medios (BIELSA \& BASSNETT, 2009; SCHÄFFNER \& BASSNETT, 2010; CRONIN, 2012; GAGNON, 2012; HERNÁNDEZ GUERRERO, 2012; o BIELSA, 2016 entre otros). Estas investigaciones remiten especialmente a la traducción periodística en la prensa, sin embargo, apenas contamos con aportaciones que se preocupan de la visibilidad o del prestigio de la persona que traduce o interpreta, o las que revelan el valor que se otorga a la actividad traductora y de interpretación en los medios: sobre la figura del intérprete leemos en DIRIKER (2005), BAIGORRI JALÓN (2012) o DEL POZO TRIVIÑO \& FERNANDES DEL POZO (2018); sobre la imagen del traductor e intérprete en la prensa española ha presentado un exhaustivo estudio EL ISLAM SIDI BAH (2015); sobre la estima del traductor editorial en la prensa se han preocupado MONTERO KÜPPER \& LUNA ALONSO (2019) y en ABEND-DAVID (2019) se ofrecen estudios sobre la representación del traductor e intérprete en los medios de comunicación. El presente trabajo, que se centra en la presencia y el prestigio de la traducción en la prensa actual, busca completar las investigaciones anteriores.

\section{Configuración del Corpus y Metodología}

El objetivo del presente estudio implica priorizar el hecho traductor como objeto frente a un análisis de la posición de quién traduce. Para la realización de esta investigación nos hemos servido

\footnotetext{
${ }^{5}$ Remitimos a la metáfora lewiniana del gatekeeping que también se cita en el ámbito de la traducción periodística, así como a la facilidad de globalización de encuadres y de conceptos metafóricos a través de calcos interculturales que ya han sido estudiado por especialistas en Comunicación o en el ámbito de la Lingüística Cognitiva, como LAKOFF \& JOHNSON (1980); ENTMAN (1993) o ARRESE (2015), en el contexto de la prensa española, y WEHLING (2016), en EUA y el ámbito germanófono.
} 
de una metodología basada en corpus y un método de investigación descriptivo-cualitativo. Para ello confeccionamos un corpus ad hoc compuesto por los textos extraídos de un periódico de ámbito estatal, El País (en lo sucesivo, EP), y otro local, La Voz de Galicia (en lo sucesivo, La Voz), ambos de corte socialdemócrata, correspondientes al lustro 2013-20176. Consideramos todos los géneros periodísticos que se encuentran en estos diarios o en los suplementos. No obstante, excluimos los blogs u otras secciones que los periódicos únicamente publican en formato digital.

Esta investigación, que se apoya en el enfoque de análisis del discurso de VAN DIJK (1985; 1995), es una aproximación global con el fin de bosquejar el discurso con respecto a las diferentes modalidades de traducción que, de una manera u otra, entran en juego en la prensa escrita. No olvidemos que el discurso (como sistema y como texto) refleja el conocimiento y la cultura de una sociedad en un momento dado (VAN DIJK, 1985, p. 10).

Estimamos oportuno centrar la atención en las manifestaciones del hecho traductor más evidentes, es decir, seleccionar aquellos textos de prensa en los que la traducción constituye la noticia o en los que la traducción es objeto de la noticia o artículo, sea como elemento constituyente, sea en calidad de causante o efecto colateral del texto ( $c f$. infra, Criterios clasificatorios). En este sentido, y también con el fin de acotar el corpus, optamos por extraer y analizar únicamente aquellos artículos que registran la palabra 'traducción' en el titular (antetítulo, título o subtítulo). Descartamos los titulares que citan un uso no traductológico del lexema 'traducción', es decir, cuando no se refiere a un trasvase lingüístico-cultural, cuando significa 'interpretación lingüística' o alude a la interpretación como actividad artística o de hechos ${ }^{7}$.

Las razones para llevar a cabo este filtrado se deben a la función especial del titular, en la que la traducción, además, se hace visible a través del propio lexema citado. El titular revela la actitud de la instancia que edita el acontecimiento y dirige la lectura, así como la recepción del artículo. Téngase en cuenta que muchos titulares, al igual que la noticia en sí, no son genuinos, sino producto de agencias de noticia. De ahí que incluso se puede llegar a crear tópicos o memes con origen en una nota de agencia, que pueden aflorar independientemente de la ideología de un periódico.

Para la obtención de los textos a partir del corpus periodístico hemos utilizado el motor de búsqueda que ofrecen los periódicos analizados en sus hemerotecas digitales. Dichas búsquedas permiten organizar los resultados por orden cronológico, pero la máscara de búsqueda no prevé

\footnotetext{
${ }^{6}$ Ambos periódicos cuentan con un amplio público lector en Galicia y España: El País (https://politica.elpais.com/) es uno de los periódicos líder de la prensa en España más leídos; en el ranking de comScore 1/2018 se sitúa en el $2^{\circ}$ lugar. https://okdiario.com/audiencia/2018/02/20/comscore-enero-2018-1850014. La Voz de Galicia (https://www.lavozdegalicia.es) es el periódico regional más leído en Galicia.

${ }^{7}$ Es frecuente confundir la práctica de la interpretación con la traducción; hecho que EL ISLAM SIDI BAH (2015) también documenta en su exhaustivo estudio.
} 
extraer selectivamente aquellos textos que únicamente incluyen la palabra 'traducción' en el titular, de manera que ha sido necesario una extracción adicional manual entre todos los textos que registrasen 'traducción` en el titulo o cuerpo del texto. La fase de extracción del subcorpus ha exigido, además, una lectura pormenorizada ${ }^{8}$ del cotexto e interpretación del contexto situacional para descubrir y determinar los valores semánticos y pragmáticos que se relacionan con la traducción.

Nos ha permitido, asimismo, entresacar los titulares que incluyen 'traducción' en las referencias de los libros traducidos reseñados, ya que hemos hecho caso omiso de las reseñas breves y extensas de traducciones editoriales cuando la traducción no es noticia9.

Siguiendo los criterios arriba expuestos, el volumen del subcorpus que hemos obtenido a partir de las noticias del quinquenio 2013-2017 de EP y La Voz es de 189 titulares, de los que más de la mitad, 112 titulares, se registraron en El Pais y 77 en La Voz.

Una vez compilado el subcorpus, procedimos a una clasificación de los textos de acuerdo con los criterios que especificamos en las siguientes líneas.

Criterios clasificatorios. Optamos por una diferenciación de dos criterios principales que nos han permitido descubrir la posición que en la prensa se otorga a la traducción en y para el sistema cultural:
A. La traducción es noticia versus la traducción es noticia colateral
B. Modalidades y ámbitos de conocimiento

\section{A. La traducción es noticia versus la traducción es noticia colateral}

Establecemos los criterios que se definen en función del discurso sobre la traducción, i.e. aquellos "textos que se convierten en ámbitos de mostración mediante las operaciones metadiscursivas, que introducen el modo de la deixis textual” (CERVERA RODRÍGUEZ, 2014, p. 79). Se atiende a los cotextos y contextos situacionales que posicionan la traducción como acontecimiento traductivo y de relevancia relativa para que se convierta en objeto de noticia (1) ${ }^{10}$. Buscamos en qué contextualización se habla de traducción sin que esta sea realmente el tema u objeto central de la noticia (ya sea en el título o en el subtítulo) posicionándose, por ende, como noticia colateral (2). Así pues, diferenciamos:

a) La traducción es noticia

\footnotetext{
${ }^{8} C f$. VAN DIJK: "ideologies of speakers or writers may be uncovered by close reading, understanding or systematic analysis" (1995, p. 135).

${ }^{9}$ En lo que a las reseñas se refiere, la traducción no suele ser noticia, sino la obra traducida. Se podría considerar que la traducción es la causante de la noticia, pues sin traducción el libro reseñado no formaría parte del sistema cultural. Así y todo, en las reseñas no se suele mencionar la traducción o únicamente se da cuenta de ella con comentarios calificativos y a menudo tipificados, como "esplendida traducción”, "fracasada traducción”, etc.

${ }^{10}$ Los números entre paréntesis remiten a los ejemplos numerados que se citan en lo sucesivo.
} 
b) La traducción es noticia colateral

Para esta clasificación hemos tenido en cuenta si la traducción es un elemento fundamental que constituye la noticia ${ }^{11}$, es decir, cuando la traducción es el acontecimiento que representa el centro de la atención informativa (a).

También hemos registrado noticias en las que la traducción es noticia colateral (b) en cuanto que puede ser causante de un acontecimiento. En este caso suele figurar en un segundo plano, en función de la línea editorial o del enfoque que le haya concedido la persona que redacta la noticia. Existe, asimismo, la posibilidad de que la traducción no sea noticia, ni noticia colateral, aunque aparezca la palabra citada en el titular como acontecimiento que gira alrededor de otros intereses, como es el caso del premio de creación en (3). Estos casos no se valoran en el estudio.

(1) Conthe dice que una buena traducción habría evitado malentendidos con las preferentes

El País (21-02-2014) EFE ECONOMÍA. El economista Manuel Conthe asegura que "una buena traducción de los términos financieros provenientes del inglés es imprescindible" y habría evitado confusiones como las producidas por las mal llamadas "participaciones preferentes", en su opinión una desafortunada traducción del inglés "preferred shares".

$(E P, 21-02-2014)$

(2) Mona Imai y Alejando Tobar logran el premio Plácido Castro de traducción Fue fallado el 3 de junio en Pontevedra y ayer se entregó en la Capela do Pilar de Corcubión

(La Voz, 27-09-2015)

(3) Guillermo Carnero gana el premio Lluis Guarner 2013

EFE 24/12/2013 - 11:58 CET

El galardón anual, dotado con más de 15.000 euros, reconoce la creación y la traducción literarias e investigación en Humanidades

(EP, 24-12-2013)

A partir de aquí, nuestra atención en el análisis se centra en las peculiaridades de la traducción y sus paratextos que se ponen de relieve de manera explícita o implícita y si estas son positivas o negativas.

\section{B. Modalidades y ámbitos de conocimiento}

\footnotetext{
${ }^{11}$ Además de la noticia propiamente dicha, en cuanto texto informativo e intencionalmente objetivo, consideramos 'noticia' los textos periodísticos de opinión e interpretativos, estimando que en este tipo de texto también se relatan noticias, si bien de manera subjetiva e incluyendo valoraciones u opiniones.
} 
En un segundo lugar, y para conocer en qué contexto se habla de la traducción, con qué se relaciona y cómo se contextualiza, clasificamos las ocurrencias de ‘traducción' según la modalidad de traducción y los ámbitos de conocimiento registrados en nuestro corpus y que enumeramos aquí:

- Traducción audiovisual (TAV)

- Traducción y TIC (TIC)

- Traducción y formación profesional (T\&FP)

- Traducción editorial (libros) (Trad. editorial)

- Traducción periodística (TP)

- Traducción en la prensa (Prensa) ${ }^{12}$

- Traducción instrumental: traducción especializada de textos técnicos, científicos, administrativos, jurídicos, etc. (Trad. instrum.)

\section{ANÁLISIS}

Antes de dar paso al análisis cualitativo, en el que ofrecemos una interpretación valorativa de los datos obtenidos, presentamos el subcorpus en términos cuantitativos. El posterior epígrafe recoge las valoraciones y los resultados de nuestro análisis descriptivo-cualitativo a partir de los titulares registrados partiendo de los criterios clasificatorios arriba esbozados con la finalidad de establecer el modo en el que se ha presentado el hecho traductor en las noticias y el objetivo de su exposición.

\subsection{Análisis cuantitativo}

La Tabla 1 resume los datos en términos cuantitativos de este corpus indicando el volumen de resultados en los que la traducción es noticia o noticia colateral, así como de su valoración global (positivo/negativo) realizada a partir de la lectura interpretativa:

Tabla 1 - Volumen de titulares en El País y La Voz de Galicia (2013-2017) en los que la traducción es noticia o noticia colateral y su valoración global

\begin{tabular}{|l|c|c|l|c|c|}
\hline \multirow{2}{*}{ Periódico } & \multicolumn{2}{|l|}{$\begin{array}{l}\text { La traducción es } \\
\text { noticia }\end{array}$} & \multicolumn{2}{l|}{$\begin{array}{l}\text { La traducción es } \\
\text { noticia colateral }\end{array}$} & Total \\
\hline & Positiva & Negativa & Positiva & Negativa & \\
\hline EP & 38 & 25 & 30 & 19 & 112 \\
\hline
\end{tabular}

\footnotetext{
${ }^{12}$ Diferenciamos la traducción periodística de la traducción en la prensa, para remitir con 'traducción en la prensa' a la traducción stricto sensu de un texto o "fuente estable" (HERNÁNDEZ GUERRERO, 2011, p.109 s.) que se traduce para ser publicado en la prensa. Con 'traducción periodística' nos referimos a la transedición (STETTING, 1989).
} 


\begin{tabular}{|l|c|c|c|c|c|}
\hline La Voz & 37 & 13 & 22 & 5 & 77 \\
\hline Total & 75 & 38 & 52 & 24 & 189 \\
\hline
\end{tabular}

La traducción como noticia en cuanto que citada en los titulares pasa prácticamente desapercibida (con excepción de la traducción literaria), si atendemos a la suma total de estos cinco años. En general, las noticias que tienen que ver con la traducción literaria suelen ser positivas, debido a que son las que aluden a premios y otros reconocimientos. La noticia suele ser negativa cuando el mensaje incide en un error de la traducción. Esto mismo sucede en el caso de las noticas colaterales en las que el hecho de que el término 'traducción' aparezca en el titular o no, no conlleva necesariamente que la traducción esté en un primer plano. La valoración negativa de la traducción en los artículos de $\mathrm{La} \mathrm{Voz}$, tanto cuando la traducción es noticia como cuando es noticia colateral, solo se registra en 18 de 77 titulares (23\%). En El País, sin embargo, esta valoración asciende a 44 de 112 titulares $(39 \%)$.

La Tabla 2 ofrece los datos en términos cuantitativos del corpus estudiado según las modalidades y ámbitos, que comentaremos en el siguiente apartado:

Tabla 2 - Ocurrencias de ‘traducción' en los titulares de El País y La Voz de Galicia (2013-2017) según modalidades y ámbitos de traducción

\begin{tabular}{|c|c|c|c|c|c|c|c|c|}
\hline & $\underset{\leftarrow}{\gtrless}$ & $\stackrel{U}{U}$ & $\frac{0}{\frac{1}{2}}$ & 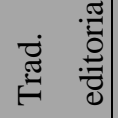 & 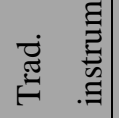 & $\stackrel{\imath}{F}$ & $\begin{array}{l}\tilde{D} \\
\text { Dू } \\
\text { D. }\end{array}$ & సٓ \\
\hline EP & 13 & 17 & 15 & 82 & 27 & - & 1 & 155 \\
\hline La Voz & 1 & 7 & 10 & 48 & 14 & 1 & - & 81 \\
\hline Total & 14 & 24 & 25 & 130 & 41 & 1 & 1 & 236 \\
\hline
\end{tabular}

El comportamiento, tanto en términos cuantitativos como cualitativos, de las publicaciones analizadas difiere. Hay una importante diferencia numérica con relación a la traducción editorial de libros. La abundancia en El País en esta modalidad se debe al suplemento cultural Babelia. Aun haciendo caso omiso de este suplemento, podemos afirmar que la mayoría de los registros que incluyen el lexema ‘traducción' en los titulares (antetítulo, titulo, subtitulo) aluden en noticias breves a traducciones de libros, especialmente de literatura traducida como novedad o como traducciones canónicas rescatadas del olvido. Únicamente 13 de los artículos registrados en La Voz y tan solo 16 en $E P$ corresponden a premios u otras celebraciones literarias. 
Tal y como presumimos, solo hemos recabado un único titular que anuncia como acontecimiento la traducción periodística (4). No obstante, es necesario leer el artículo para descubrir que se trata de un error de traducción de la agencia Reuters, ya que el titular no lo delata:

(4) El error de traducción del director de Roomba que costó millones de dólares Después de que saltara la noticia de que la compañía iba a vender los mapas de las casas de sus clientes, las acciones de la compañía se dispararon en la Bolsa. Pero la noticia se debía en realidad a un error de traducción

(La Voz, 24-08-2017)

Figura, asimismo, un único resultado que manifiesta de forma visible el hecho traductor stricto sensu (5):

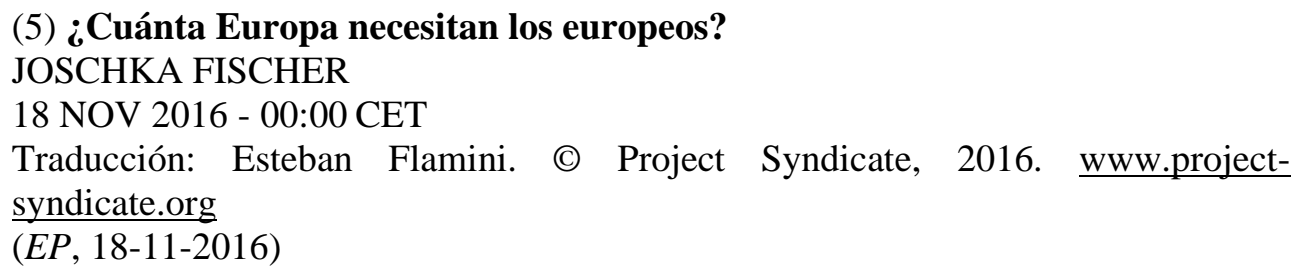

Si tenemos en cuenta la proporcionalidad de los dos periódicos, la traducción instrumental goza, a nuestro modo de ver, de más visibilidad, en comparación con las otras modalidades y ámbitos (con excepción de la traducción editorial) y se convierte en noticia sobre todo cuándo resulta ser una fuente de incordio. La temática que gira alrededor de la formación o profesión figura en menos ocasiones en el titular. Los titulares que registran las traducciones audiovisuales parecen ser un reflejo de la conciencia que se tiene en la sociedad española del trasvase lingüístico-cultural que acompaña esta actividad. Pero aquí La Voz no destaca este aspecto en los titulares.

\subsection{Análisis cualitativo}

\subsubsection{La traducción es noticia versus la traducción es noticia colateral}

\section{a. La traducción es noticia}

Cuando la traducción aparece en el titular, podríamos deducir que siempre es noticia $(6 ; 7)$. No obstante, esto no siempre es así, y, además, hemos computado varios casos en los que el término aparece en el antetítulo o subtítulo, dándole protagonismo a un aspecto secundario de la noticia que funciona como reclamo, aunque la traducción resulta ser la noticia (8):

(6) El futuro de la traducción, ¿los robots? ¿Literalidad o sentido? ¿Mecanización o estilo? Las nuevas tecnologías plantean muchas dudas al respecto de cómo debe ser el futuro de la traducción (EP,Tecnología, 26-08-2017) 
(7) El Marco plantea el papel de la traducción como factor creativo

La propuesta del suizo Marco Waldmeier fue la ganadora del premio de jóvenes comisarios.

(La Voz (Vigo), 29-05-2015)

(8) «Flaubert era un obseso del estilo»

Armiño publica una traducción de «Madame Bovary» con pasajes inéditos

(La Voz 17-08-2014)

En el ejemplo (8) se aprecia cómo se priva a la traducción de protagonismo en el titular, optando por un elemento sensacionalista para el título. Solo en el subtítulo se ofrece la información que realmente es objeto de la noticia y que además se desenvuelve en el texto. Generalmente, a este tipo de traducción no se concede el debido protagonismo, ya que prevalece la autoría o la propia publicación de una novedad editorial. De hecho, constatamos que aún en la actualidad no se le atribuye a la traducción de obras el valor que merece si atendemos al espacio que ocupa en las páginas culturales, de sociedad o locales. Una realidad que pocas veces se transmite verbalizándola como sí se hace en el ejemplo (9):

\section{(9) Hablar castellano en todos los idiomas}

Solo nos acordamos de los traductores cuando nos dicen que 'El gran Gatsby' y 'Madame Bovary' deberían titularse 'Gatsby el Magnífico' y 'La señora Bovary' $(E P, 04-11-2015)$

La traducción se presenta como noticia de importancia especialmente cuando se estima escandalosa, enfatizando así en el valor negativo (10; 11):

\section{(10) Traducciones erróneas}

Reproches a propósito de algunas traslaciones equivocadas, carentes de sentido o fluidez, y por el empleo de términos en inglés $(E P, 02-06-2013)$

\section{(11) Lo que cuesta el 'billion' que no es billón}

La traducción a 23 idiomas multiplica la factura mientras una 'eurolengua' se abre paso

$(E P, 23-04-2013)$

La traducción que se cita en el título como noticia, no siempre es negativa ni resultado de un escándalo profesional o de malas prácticas. También se realza la traducción que genera un beneficio económico incluso importante $(12 ; 14)$, aunque en nuestro corpus este énfasis es ocasional:

(12) La primera traducción al chino de El Quijote traerá miles de turistas a Alcalá 
Uno de los principales touroperadores de China en España, nieto del traductor de la obra, firma un convenio para traer más visitantes a la ciudad $(E P, 21-01-2016)$

(13) Attesor: la agencia de traducción del futuro

Nace en A Coruña el primer «coworking» de traductores de Galicia con una red de más de mil profesionales capaz de traducir a todos los idiomas del mundo

(La Voz, 30-04-2017)

\section{(14) Nada de perder el tiempo}

Milena Busquets autora de 'Todo esto pasará' cuyo manuscrito despertó gran expectación en la Feria de Frankfurt y ha firmado contratos de traducción en más de 30 países

$(E P, 06-07-2015)$

Cuando la noticia sale de la sección habitual de libros y pasa a visibilizarse en la de mercados (12 -14), la traducción adquiere un valor añadido y, en consecuencia, la empresa gana en repercusión comparable a la dimensión de las que forman parte de grandes grupos.

\section{b. La traducción es noticia colateral}

La traducción es noticia colateral cuando se presenta como tal en el subtítulo, y, como consecuencia, permanece en un segundo plano. En varios ejemplos registrados, la traducción es una noticia derivada de otros hechos informativos, tratándose por lo tanto de una noticia colateral, incluso si el hecho traductor es la razón desencadenante de la misma $(15 ; 16)$ :

\section{(15) La burocracia salva a los Kinahan}

La causa contra la mafia irlandesa naufraga por retrasos de 18 meses en la traducción de documentos y trámites sin resolver tras seis años

$(E P, 04-11-2016)$

\section{(16) Polanski comparece en el juicio por su extradición}

El tribunal todavía no decidirá sobre la entrega del director a las autoridades de EE UU. El proceso se demorará porque es necesaria la traducción de documentación $(E P, 25-02-2015)$

Incluso en contextos de premios de traducción esta puede quedar relegada a un segundo plano ya que se realza el premio o gana protagonismo otro aspecto que se considera más importante y se posiciona como insólito $(17 ; 18)$ :

(17) Un 'Edipo' audaz y potente para el lector del siglo XXI

El filólogo presenta en un libro su nueva traducción de la tragedia de Sófocles y un detallado ensayo

(EP, 03-04-2013) 
(18) Cultura anula el Premio Nacional a la Mejor Traducción por error del jurado

Sí se mantiene la validez del concedido a Ramón Buenaventura, el Premio Nacional a la Obra de un Traductor

(La Voz, 04-11-2016)

\subsubsection{Modalidades y ámbitos de conocimiento}

Analizamos y valoramos en las siguientes líneas cómo se presenta la traducción a la sociedad y qué cualidades se le atribuyen a la traducción en las noticias de nuestro corpus en función de la modalidad. Los títulos de los apartados $a$ ) - e) identifican los valores adjudicados y temáticas evocadas más reveladores registrados en el corpus de los titulares.

\section{a) La traducción literaria genera beneficio económico y cultural}

Desde el punto de vista cualitativo, en relación a la traducción de libros como noticia, destaca que se realza sobre todo literatura traducida como novedad o como traducciones canónicas recuperadas (19). También son muy significativos los casos en los que la noticia viene derivada de la obtención de algún reconocimiento (23):

\section{(19) Una traducción para sacar a Dumas del purgatorio}

Una nueva edición de 'El conde de Montecristo' revaloriza la novela (EP, 23-12-2017)

(20) Salvador Peña Martín y Malika Embarek, Premios Nacionales de Traducción 2017

El docente recibe el galardón a Mejor Traducción y la filóloga a la Obra de un Traductor

(EP, 26-10-2017)

En el siguiente ejemplo (21) se señala el valor cultural de la publicación contribuyendo así a la revalorización del sistema cultural en cuestión dentro del espacio ibérico. Se destaca sobre todo el beneficio cultural y no la acumulación de capital económico (inmediato) de la traducción literaria. Aun así, hay algunas noticias que llaman la atención sobre el beneficio de una buena traducción literaria y la ponen en relación con la economía como sector estratégico (22):

(21) A tradución de «Ilustrísima» en inglés proxecta internacionalmente a obra de Casares

O Gaiás homenaxea o autor das Letras Galegas cunha exposición centrada na súa producción literaria, de investigador e tamén tradutor

(La Voz, 16-05-2017)

(22) La importancia de la calidad en la traducción 
Los textos traducidos tienen una influencia directa en la economía $(E P, 29-09-2014)$

\section{b) La traducción instrumental causa problemas}

La traducción instrumental es, en el corpus analizado, la menos estimada: la traducción de textos no destinados a la edición suele impedir o dificultar el funcionamiento de la justicia o es una inversión económica que complica el avance de los procesos industriales, etc. Se trata normalmente de una traducción necesaria pero que solo se vuelve visible cuando causa un problema. Los ejemplos en los que el mensaje es positivo son escasos $(25 ; 26)$ :

(23) La traducción al español frena la causa de la redada de O Vao Un abogado madrileño solicitó la traslación de los autos en gallego (La Voz, 02-08-2013)

(24) Un anuncio ambiguo del PP anima a Rajoy a marcharse

La traducción al euskera del lema 'A favor' por 'Alde' se puede interpretar como "vete" o "fuera" $(E P, 13-06-2016)$

\section{(25) La copia también es original} PABLO DE LLANO

La editorial independiente Alias convierte la traducción de textos de arte en un ejercicio de reinterpretación con sello propio

(EP, 24-04-2015)

(26) Covasolutions da soporte a las empresas españolas que dan el salto a EE.UU. Galicia, el mejor entorno

Esther Diz transformó su empresa de traducción en un servicio integral para la internacionalización

(La Voz, 31-03-2015)

\section{c) Traducir para la pantalla exige habilidades}

La traducción que se realiza para la pantalla ocupa un espacio no desdeñable en la prensa, quizás cada vez con más intensidad debido a las (para muchas personas) aun nuevas formas de consumir productos audiovisuales (series, cine, videojuegos, etc.). Aun así, en nuestro corpus encontramos menos incidencias de las esperadas. Las noticias sobre esta modalidad tratan sobre todo de la dificultad de la traducción de juegos de palabras, nombres propios que tienen distinto significado o diferente connotación cultural, del humor, y de la problemática de la traducción que pone en contacto dos culturas más o menos cercanas.

\section{(27) La difícil traducción del humor}


Conseguir que Homer Simpson sea gracioso también en español o mantener los juegos de palabras de series como 'Modern Family' es un reto para los traductores

$(E P, 18-01-2017)$

(28) Así se resolverá ese problema con el doblaje en 'Juego de tronos'

El capítulo 'El portón' plantea un dilema de traducción imposible de resolver $(E P, 25-05-2016)$

\section{d) Traducción y TIC - una utilidad cuestionada}

A pesar de que cada vez se haga más uso de las TIC para traducir, su presencia explícita en nuestro corpus es escasa. La imagen que se transmite de la traducción realizada mediante el uso de las nuevas tecnologías no siempre es unívoca y vacila entre útil o práctica y conflictiva. Se puede percibir algún escepticismo en noticias que a priori son positivas y parecen una solución exitosa:

\section{(29) El nuevo traductor del euskera al castellano se puede usar en el móvil}

El sistema permitirá también volcar textos en inglés a la lengua vasca (EP, 02-04-2014)

(30) Cuando «o grelo» se convierte en «el clítoris»

Los vecinos de As Pontes se sorprendieron al ver que su tradicional Feira do grelo se transformaba por obra del traductor de Google en Feria clítoris (La Voz, 31-10-2015)

\section{(31) La tecnología que puede sustituir a los idiomas}

Los sistemas de traducción instantánea se empiezan a popularizar en reuniones de negocios y ONG, pero aún están lejos de sustituir a los humanos $(E P, 01-12-2017)$

Aun cuando el error de la traducción automática es el factor causante de la noticia (30), esta se puede presentar como una irrisoria anécdota. La noticia sobre la intervención de la máquina en la tarea de la persona que traduce no siempre es negativa, pero sí lo es, cuando la máquina actúa sola sin pasar por el filtro revisor del cerebro humano. Se informa que las nuevas tecnologías ayudan en la tarea de la traducción, pero no sustituyen a la persona que traduce, y es importante que esto se sepa. Es decir, el discurso (aquí explícito) apunta la actividad traductora como tarea compleja que no se resuelve con una máquina:

(32) Pilot: el traductor instantáneo que nos hace más tontos

El aparato, que permite la comunicación en todas las lenguas, hará que todo gire aún más en torno a nuestro propio mundo

$(E P, 12-03-2017)$ 
(33) Un palestino detenido por un error de traducción de Facebook El algoritmo reprodujo la expresión "atacadles" en hebreo cuando había escrito "buenos días" en árabe en su página de la red social $(E P, 25-10-2017)$

\section{(34) Los profesionales de traducción recelan del traductor de Google}

Los profesionales de la Traducción denunciaron ayer el intrusismo durante la celebración de su patrón, San Jerónimo, en la facultad de Traducción de la Universidade de Vigo.

(La Voz, 02-10-2013)

Las noticias dispares y muy ocasionales en los medios analizados, en cuanto al valor de las TIC para la traducción, y la estimación de las traducciones realizadas por nuevas tecnologías, muestran un parco interés en ilustrar sobre el funcionamiento de una herramienta que forma parte del día a día en los medios de comunicación. Pero son precisamente las traducciones periodísticas automatizadas, las noticias realizadas por un robot periodista, las que pueden ser causante de noticias falsas que causan revuelo o incluso pueden desembocar en un "deadliest error" (ZANETTIN, 2016). Estas traducciones periodísticas no suelen ser objeto de noticia; de hecho, en el corpus no consta ningún ejemplo.

e) Noticias sobre aspectos profesionales en traducción

Estas noticias suelen valorizar la profesión en cuanto oficio que requiere de competencias además de conocimientos y habilidades específicas. Atendiendo a lo comentado sobre la traducción periodística o para la prensa, que más bien se parece ningunear, a priori resulta sorprendente que se pongan en valor aspectos de la formación y la actividad profesional.

(35) ¿Quieres trabajar en Netflix? Haz este test

La empresa recluta a los expertos en traducción con una prueba 'online' $(E P, 18-03-2017)$

(36) UN SIGLO DE 'EN BUSCA DEL TIEMPO PERDIDO'

'En busca del tiempo perdido', la traducción de nuestras vidas

Tres traductores de Marcel Proust relatan su experiencia al llevar 'En busca del tiempo perdido' al castellano

(37) (La Voz, 23-06-2013)

Miguel Sáenz: «La traducción es tan antigua como la prostitución, pero peor pagada»

$(E P, 13-11-2013)$ 


\section{Conclusiones}

En los apartados anteriores hemos analizado aspectos denotativos y connotativos para delimitar el protagonismo de la traducción e identificar elementos metatraductivos a partir del análisis semántico-pragmático de los titulares que configuran el subcorpus. Dicho análisis nos ha permitido realizar una aproximación al relato presente en la prensa de la traducción en cuanto noticia. El discurso sobre la traducción se revela a través de los contenidos relatados en el contexto y cotexto (cf. 3.2.). A partir de estos elementos o posicionamientos registrados se compone una idea o imagen de la traducción que determina su valor para la sociedad.

El análisis semántico nos ha permitido conocer los significados explícitos: los valores positivos se revelan en palabras como beneficio, bueno o premio; y los lexemas equivocado, erróneo, problema o naufragar nos descubren un valor claramente negativo. El significado pragmático, que se encuentran en estrecha relación con el contexto social y cultural de los acontecimientos, puede dar lugar a implicaturas imprecisas (cf. los ejemplos 29-31), ya que el discurso también se constituye a través de la recepción.

En cuanto al periodo analizado (2013-2017), podemos concluir que la traducción es noticia o noticia colateral en muy pocas ocasiones por lo que cuenta con una escasa visibilidad: a menudo se trata, además, de una noticia derivada de otros hechos informativos, que hemos denominado "noticia colateral", pues se le da más importancia a otro aspecto del acontecimiento que se relata. Constatamos que en muchos casos, la traducción, aun siendo causante de la misma, se presenta como noticia colateral. Con todo, los resultados muestran que la valoración de la traducción en la prensa estudiada es mayoritariamente positiva (127 de los 189 titulares, i.e. $68 \%$ ).

Los datos rescatados revelan un volumen superior de alusiones (sobre todo positivas) a la traducción editorial, especialmente la literaria, aunque sin profundidad crítica y casi siempre en formato de noticia breve. Observamos que existe un bajo número de noticias que tratan de la traducción instrumental o de la intervención de las TIC que mantienen un discurso negativo. En este sentido, el discurso transmitido sobre la traducción es que la actuación del ser humano resulta imprescindible para la traducción. Y, si bien se trata de contadas manifestaciones, se concibe y presenta como tarea compleja.

En relación con las noticias registradas y según la modalidad de la traducción podemos diferenciar entre un discurso en el que la traducción se asocia con algo beneficioso o bien supone una actividad que implica un inconveniente. Esta idea no se altera a lo largo del periodo estudiado.

El análisis nos permite esbozar los siguientes relatos:

- La traducción (editorial / literaria) genera capital cultural. 
- La traducción genera capital económico (ocasionalmente).

- La traducción exige destrezas (profesión y formación).

- La traducción instrumental es fuente de problemas.

- La traducción no siempre cumple su función comunicativa.

El discurso que prevalece en términos cuantitativos y cualitativos es aquel que se transmite de manera generalmente explícita sobre la traducción literaria y ensayística pues se caracterizan como beneficio para la sociedad, en términos de acumulación de capital cultural, de relevancia para el porvenir de la propia comunidad.

El valor económico añadido no se suele asociar a la traducción, lo que también se debe a que la mayoría de las noticias (colaterales) la presentan precisamente como producto cultural cuyo rendimiento económico no se considera de relevancia. Así y todo, no se ha de obviar que la cultura también es sector estratégico cultural y como tal forma parte del engranaje industrial.

A pesar de tratarse de aspectos muy puntuales que se valoran en el corpus examinado, consideramos la mera inclusión de este tipo de noticias (que de por sí son positivas) un discurso sobre la traducción doblemente positivo a favor de la visibilidad de la traducción.

Para finalizar, recordaremos que la prensa y los medios de comunicación en general tienen por cometido principal informar, pero también la responsabilidad de formar a la sociedad a la que se dirigen. La imagen que se transmite en los titulares de la prensa sobre la traducción es relevante no solo para la Traducción como campo de estudio, sino también para la opinión pública que se encuentra en estrecha dependencia del llamado poder publicativo. Nuestro estudio contribuye en este sentido a saber qué valor se transmite cuando en la prensa se habla de traducción.

El discurso sobre la traducción periodística ayuda a visibilizar y concienciar sobre la existencia de un proceso traductor inherente a la producción de un importante porcentaje de noticias $\mathrm{y}$, en consecuencia, de un filtro (gatekeeper) que encauza la ideología y la recepción de un acontecimiento relatado.

En cuanto a la traducción periodística, el análisis del corpus deja patente que el discurso traductivo está prácticamente ausente, pese a que, como decíamos al inicio de este estudio, la traducción en este espacio de comunicación global es prácticamente consustancial a los medios. En aras de una mayor transparencia y objetividad queda, pues, pendiente, volver visible la traducción periodística a través de un encuadre explícito.

\section{BIBLIOGRAFÍA}

ABEND-DAVID, D. (ed). Representing Translation. USA: Bloomsbury Publishing, 2019. 
ARRESE, Á. Las metáforas de la crisis del euro en la prensa española. Communication \& Society, Pamplona, v. 28, n. 2, p. 19-39, 2015.

BAIGORRI JALÓN, J. La imagen caleidoscópica del intérprete. Algunos ejemplos de la prensa anglosajona de los siglos XIX y XX. In: CRUCES COLADO, S. et al. (eds.) Traducir en la frontera. Granada: Atrio, 2012, p. 229-44.

BASTIN, G. \& ITURRIZA, M. ${ }^{a}$ G. La traducción como elemento creador de identidad en la prensa independentista de Venezuela (1808-1822). TRANS, Málaga, 12, p. 81-94, 2008.

BIELSA, E. La traducción en los medios de comunicación: una perspectiva cosmopolita. In:

MARTÍN RUANO, M. R.; A. VIDAL CLARAMONTE (ed.) Traducción, medios de comunicación, opinión pública. Granada: Comares, 2016, p. 17-35.

BIELSA, E. \& BASSNETT, S. Translation in Global News. Londres etc.: Routledge, 2009.

CERVERA RODRÍGUEZ, Á. Análisis lingüístico-pragmático de titulares de artículos de opinión.

Lingüística y Literatura, Medellín, n. 66, p. 65-88, 2014. Disponible en:

https://aprendeenlinea.udea.edu.co/revistas/index.php/lyl/article/view/20212/17101. Acceso: 02 mayo 2018.

CRONIN, M. Translation in the Digital Age. London, etc.: Routledge, 2012.

DEL POZO TRIVIÑO, M. \& FERNANDES DEL POZO, D. Estudio de la reputación online de la profesión de intérprete en la prensa digital española: primeras aproximaciones. (Inédito), presentado en III XESCOM, Simposio Internacional sobre Gestión de la Comunicación. A Coruña: Universidade da Coruña, 2018.

DIRIKER, E. Presenting simultaneous interpreting: Discourse of the Turkish media, 1988-2003. aiic.net, March 21, 2005. Disponible en: http://aiic.net/p/1742. Acceso: 12 mayo 2019.

EL ISLAM SIDI BAH, N. La prensa y la traducción e interpretación en los servicios públicos. Madrid: Universidad Autónoma de Madrid, 2015. Tesis doctoral inédita. Disponible en: https://repositorio.uam.es/bitstream/handle/10486/669535/el_islam_ould_sidi_nour.pdf?sequence= 1. Acceso: 05 mar. 2018.

ENTMAN, R. Framing: Towards a Clarification of a Fractured Paradigm. Journal of Communication, Nueva York, etc., v. 43, n. 3, p. 51-58, 1993.

GAGNON, C. La visibilité de la traduction au Canada en journalisme politique : mythe ou réalité ? Meta, Montreal, v. 57, n. 4, p. 943-59, 2012.

HERNÁNDEZ GUERRERO, M. J. Traducción y periodismo. Bern: Peter Lang, 2000.

HERNÁNDEZ GUERRERO, M. J. Presencia y utilización de la traducción en la prensa española. Meta, Montreal, v. 56, n. 1, p. 101-118, 2011.

HERNÁNDEZ GUERRERO, M. J. La traducción al servicio de una línea editorial: la primavera árabe en el diario El País. Meta, Montreal, v. 57, n. 4, p. 960-76, 2012.

LAKOFF, G. \& JOHNSON, M. Metaphors we live by. Chicago: The University Press, 1980.

MARTÍN RUANO, M. R.; SALES VIDAL, C Identidades en traducción: representaciones transculturales de las subjetividades colectivas en los medios. Debats, Valencia, n. 121, p. 84-93, 2013.

MONTERO KÜPPER, S.; A. LUNA ALONSO. The Public Image of Book Translators in the Digital Press. In: TÚÑEZ-LÓPEZ, M. et al. (coord.) Communication: Innovation \& Quality. Cham: Springer, 2019 (publicado en línea 2018), p. 313-28. 
SCHÄFFNER, CH. \& BASSNETT, S. (eds.). Political Discourse, Media and Translation. Newcastle upon Tyne: Cambridge Scholars Publishing, 2010.

STETTING, K. Transediting - A new term for coping with the grey area between editing and translating. In: CAIE, G. et al. (eds) Proceedings from the Fourth Nordic Conference for English Studies. Copenhaguen: K $\phi$ benhavns Universitet, 1989, p. 371-82.

VALDEÓN, R. A. (ed.) Journalisme et traduction. Meta, Montreal, v. 57, n. 4, 2012.

VALDEÓN, R. A. Fifteen years of journalistic translation research and more. Perspectives, Abington, v. 23, n. 4, p. 634-62, 2015.

VALDEÓN, R. A. Traducción periodística y gatekeeping. In: MARTÍN RUANO, M. R.; VIDAL CLARAMONTE, A. (ed.). Traducción, medios de comunicación, opinión pública. Granada: Comares, 2016. p. 35-5.

VAN DIJK, T. A. Introduction: Levels and dimensions of discourse analysis. In: VAN DIJK, T. A. (ed.) Handbook of Discourse Analysis. London: Academic Press, 1985. p. 1-11. v. 2.

VAN DIJK, T. A. Ideological discourse analysis. In: VENTOLA, E.; SOLIN, A (ed.). The New Courant, v. 4, n. Spec. Interdisciplinary approaches to Discourse Analysis, Helsinki, p. 135-161, 1995.

VAN DOORSLAER, L.Translating, Narrating and Constructing Images in Journalism with a Test Case on Representation in Flemish TV News. Meta, Montreal, v. 57, n. 4, p. 1046-59, 2012.

WEHLING, E. Politisches Framing: Wie eine Nation sich ihr Denken einredet und daraus Politik macht. Köln: Halem, 2016.

ZANETTIN, F. 'The deadliest error': translation, international relations and the news media, The Translator, Manchester, 22:3, p. 303-18, 2016. Disponible en https://doi.org/10.1080/13556509.2016.1149754. Acceso: 16 nov. 2018. 